\title{
Diamond Films for Implantable Electrodes
}

\author{
Petra Henychová, Klára Hiřmanová, Martin Vraný \\ Department of Biomedical Technology, Faculty of Biomedical Engineering, Czech Technical University, Kladno, Czech \\ Republic
}

Corresponding author: petra.henychova@fbmi.cvut.cz

\begin{abstract}
Diamond is a promising material for implantable electrodes due to its unique properties. The aim of this work is to investigate the growth of boron-doped nanocrystalline diamond (B-NCD) films by plasma-enhanced microwave chemical vapor deposition at various temperatures, and to propose optimal diamond growth conditions for implantable electrodes. We have investigated the temperature dependence $\left(450^{\circ} \mathrm{C}-820^{\circ} \mathrm{C}\right)$ of boron incorporation, surface morphology and growth rate on a polished quartz plate. Surface morphology and thickness were examined by atomic force microscopy (AFM). The quality of the films in terms of diamond and non-diamond phase of carbon was investigated by Raman spectroscopy. AFM imaging showed that the size of the grains was determined mainly by the thickness of the films, and varied from an average size of $40 \mathrm{~nm}$ in the lowest temperature sample to an average size of $150 \mathrm{~nm}$ in the sample prepared at the highest temperature. The surface roughness of the measured samples varied between $10\left(495^{\circ} \mathrm{C}\right)$ and $25 \mathrm{~nm}\left(800{ }^{\circ} \mathrm{C}\right)$. The growth rate of the sample increased with temperature. We found that the level of boron doping was strongly dependent on temperature during deposition. An optimal B-NCD sample was prepared at $595^{\circ} \mathrm{C}$.
\end{abstract}

Keywords: Nanocrystalline diamond, implantable electrode.

\section{Introduction}

An implantable electrode is an invasive electronic device, and for this reason there are very high requirements on the materials that are used. Long-term functionality and good performance must be ensured by selecting appropriate materials for the electrode [4]. Above all, the electrodes must not adversely affect the health of the patient [1]. The material of the implant must be biocompatible, and must not cause an allergic reaction [4]. There should be no acute or chronic adverse reaction, and no evidence of toxicity. In addition, the host organism must not affect the implanted system, and the system must preserve its properties in the aggressive environment of a living organism [1]. The impedance between electrode and tissue must be low and stable in order to provide reliable measurements or stimulations [4]. Electrodes can be exposed to potentials of hundreds of volts: the material must be resistant to electrochemical degradation [5]. Last but not least, the material should be compatible with magnetic resonance [4]. Further important factors are manufacturability, reproducible quality, and cost [1]. Diamond is a promising material for implantable electrodes due to its unique properties. Most importantly, diamond is biologically compatible. It is the hardest material in nature. It has extreme wear resistance and resistance to chemical corrosion. It is a great thermal conductor, and its thermal expansion coefficient at room temperature is very low. Diamond can easily be doped, and then it becomes a semiconductor. It does not melt during current flow, and it has a wide potential window [2].

Diamond can be made in many different ways. One example is the high-pressure high-temperature (HPHT) technique. This method simulates the conditions in which diamonds are formed in nature. In the nature, diamond is formed deep underground, under very high pressure and temperature. This results in the formation of diamond monocrystals. Diamond in form of monocrystals is not a convenient material for most medical applications. Another commonly used technique for diamond deposition is CVD (chemical vapor deposition). This method enables not only single crystalline diamonds to be deposited. Carbon atoms are added from the gas phase to the initial template. Lower pressure and temperature is used. CVD is a more economical method. The biggest advantage is that a very thin layer of diamond is formed [2].

Platforms for implantable diamond electrodes are made in several steps. A thin diamond film is grown on a silicon wafer. The second step is deposition of a film of a suitable polymer (e.g. poly (methyl methacrylate) or polyimide) by spin coating followed by hardening of the polymer by baking. Chemical etching of the silicon wafer leaves a polycrystalline diamond film on a flexible substrate.

In this paper, we focus on optimizing the growth of thin diamond films by plasma-enhanced microwave chemical vapor deposition. We investigate the temperature dependence of boron incorporation, surface morphology and growth rate. We propose optimal diamond conditions for application in implantable electrodes. 


\begin{tabular}{cccc}
\hline Run ID & $\begin{array}{r}\text { Temperature } \\
\left({ }^{\circ} \mathrm{C}\right)\end{array}$ & $\begin{array}{r}\text { Pressure } \\
(\mathrm{mBar})\end{array}$ & $\begin{array}{c}\text { Power } \\
(\mathrm{W})\end{array}$ \\
\hline $\mathrm{S} 820$ & 820 & 90 & 1522 \\
\hline $\mathrm{S} 670$ & 670 & 50 & 1150 \\
\hline $\mathrm{S} 595$ & 595 & 50 & 750 \\
\hline $\mathrm{S} 450$ & 450 & 50 & 550 \\
\hline $\mathrm{S} 490$ & 490 & 50 & 650 \\
\hline
\end{tabular}

Table 1: Variable conditions for MWPECVD

\section{Experimental}

The study was performed for five different substrate temperatures during deposition. During the study, the temperatures ranged between $450{ }^{\circ} \mathrm{C}$ and $820^{\circ} \mathrm{C}$. The growth conditions and temperatures are summarized in Tab. 1.

\section{$2.1 \quad$ Substrate pretreatment}

We used a polished quartz plate $10 \times 10 \times 1 \mathrm{~mm}$ (MaTecK GmbH, Germany) as the substrate.

The substrates were cleaned using isopropyl on dust-free cellulose wadding. Then the sample was inserted into deionized water $(2 \mathrm{M} \Omega \mathrm{m})$ and sonicated in an ultrasonic bath at $80^{\circ} \mathrm{C}$ for 10 minutes. Finally, we dried the substrate with dry compressed air.

Initial nucleation of the substrates were done by immersion in a solution of NanoAmando nanodiamond (nanocarbon Research Institute, Japan) at a concentration of $0.2 \mathrm{~g} / \mathrm{l}$, followed by spin coating at $3800 \mathrm{rpm}$ for $30 \mathrm{~s}$. The declared size of the nanodiamonds in the solution was $5 \pm 0.9 \mathrm{~nm}$.

\subsection{Growth of NCD}

NCD was grown by the microwave plasma enhanced chemical vapor deposition method (MWPECVD). We used an ASTeX AX5010 reactor (Seki Technotro Corp., Japan).

All samples were grown for 90 minutes with a fixed boron concentration in a gas mixture (2000 ppm $\mathrm{B} / \mathrm{C})$, and there was a fixed concentration of methane $(0.5 \%)$. The variable growth conditions are presented in Tab. 1.

\subsection{Characterization}

The surface roughness and thickness of the layer was obtained by atomic force microscopy (AFM). The roughness (Root Mean Square) is calculated as

$$
R_{q}=\sqrt{\frac{1}{n} \sum_{i=1}^{n} y_{i}^{2}}
$$

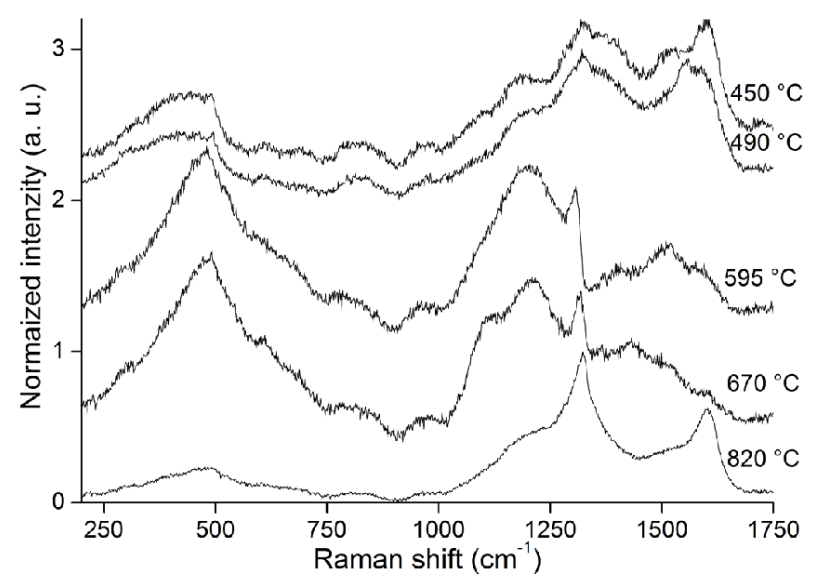

Figure 1: Raman spectra (excluding $633 \mathrm{~nm}$ ) of boron-doped NCD films prepared at various temperatures. Peaks at $500 \mathrm{~cm}^{-1}$ and $1225 \mathrm{~cm}^{-1}$ related to boron doping are clearly visible for the samples prepared at $595^{\circ} \mathrm{C}$ and $670^{\circ} \mathrm{C}$.

This is analogous with standard deviation in statistics.

We used an NTEGRA Prima NT MDT microscope and Budget Sensors Tap AI-150G tips at a frequency of $150 \mathrm{kHz}$ and stiffness of $5 \mathrm{~N} / \mathrm{m}$. For the thickness measurements, a part of the substrate was cleared of seeds prior to growth.

This resulted in an area without diamond growth, which was used as a reference for the thickness evaluation. The growth rate was calculated by dividing thickness by growth time.

Layer quality was measured using an INVIA Raman microscope. Spectra were taken using a laser power of $2 \mathrm{~mW} 633 \mathrm{~nm}$ at room temperature. All spectra were normalized to the characteristic spade at diamond wave number around $1332 \mathrm{~cm}^{-1}$.

The temperature during deposition was measured using a Pyrometric 92 two-color pyrometer (Williamson, USA).

\section{Results and discussion}

\subsection{Raman spectroscopy}

All samples exhibit a peak between $1306 \mathrm{~cm}^{-1}$ and $1322 \mathrm{~cm}^{-1}$ attributed to diamond ( $\mathrm{sp}^{3}$ bonds). The position intensity of the diamond peaks is influenced by many factors, including the number of $\mathrm{sp}^{3}$ bonds in the material and the level of boron doping. High boron doping shifts the diamond Raman peak downwards and reduces its intensity [6]. Another prominent peak in all spectra is at $500 \mathrm{~cm}^{-1}$. This peak is related to the presence of boron in the crystal lattice of diamond.

The intensity of this peak can be used for a semiquantitative estimate of the boron content [3]. Fano resonance, another spectral feature related to the presence of boron, is also distinguishable in all spectra. 


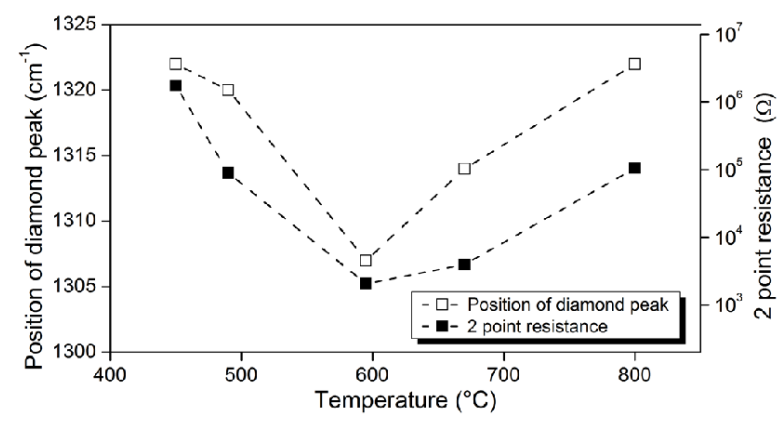

Figure 2: Position of the diamond Raman peak and resistance measured by the 2 -point probe method.

The relative intensity of the peaks varies among the investigated samples. According to the Raman spectra, the highest boron incorporation was achieved at a temperature of $595^{\circ} \mathrm{C}$, followed by a temperature of $670^{\circ} \mathrm{C}$. These samples feature an intense peak at $500 \mathrm{~cm}^{-1}$, prominent Fano resonance at $1225 \mathrm{~cm}^{-1}$, and a shifted diamond Raman peak.

The sample prepared at $820^{\circ} \mathrm{C}$ has a narrow Raman peak, and boron related peaks were less apparent.

The spectra recorded for samples prepared at $450{ }^{\circ} \mathrm{C}$ and $490^{\circ} \mathrm{C}$ are similar to each other. Both have a broad diamond Raman peak of low intensity, indicating small grains and low quality of the diamond. The intensity of the peaks related to boron is similar to the results for the sample prepared at $820^{\circ} \mathrm{C}$.

Raman spectroscopy also shows the presence of a high amount of non-diamond phase in some samples. Most notably, the $\mathrm{G}$ band $\left(1580 \mathrm{~cm}^{-1}\right)$ that appears in the spectra of the B-NCD samples is attributed to crystalline graphite. This peak is relatively strong in the samples prepared at the lowest temperature $\left(450{ }^{\circ} \mathrm{C}\right)$ and at the highest temperature $\left(820^{\circ} \mathrm{C}\right)$. It should be noted that the laser excitation that we used is very sensitive to $\mathrm{sp}^{2}$ bonding.

To verify our assumption, based on Raman spectroscopy, that there is higher boron doping for the sample prepared at temperature $595^{\circ} \mathrm{C}$, we measured the resistance of the films by a simple 2-point probe method. The results adjusted for the thickness of the film are plotted in Fig. 2. Although 2-point probe measurements allow only a crude estimate of the true resistivity of the sample, Fig. 2 clearly shows a correlation between the resistance and the position of the diamond Raman peak, which is influenced by the presence of boron in the material. The higher resistance of films prepared at lower temperatures may be caused by the fact that the films are not completely continuous.

In summary, all of the B-NCD films exhibit evidence of boron doping. There is a lower level of boron doping for low temperatures. The level then increases with temperature, reaching a maximum at around $600^{\circ} \mathrm{C}$, and then decreases again for high temperatures.

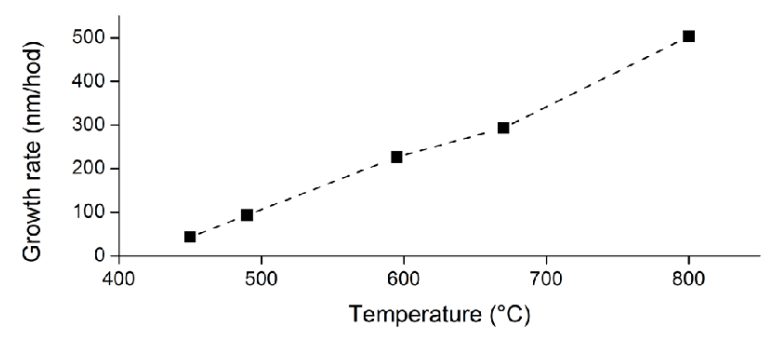

Figure 3: Growth rate of a B-NCD film as a function of temperature.

\subsection{Growth rate and surface morphology}

AFM imaging showed that the B-NCD films (Fig. 4) consist of randomly-oriented grains of submicron size. The size of the grains was mainly determined by the thickness of the films, and varied from an average size of $40 \mathrm{~nm}$ in the lowest temperature sample to an average size of $150 \mathrm{~nm}$ in the sample prepared at the highest temperature. For lower temperature, we observe small grains without visible facets. Clearly visible crystal facets can be identified on the samples prepared at $595{ }^{\circ} \mathrm{C}$ and above. The surface roughness of the measured samples varies between $10 \mathrm{~nm}\left(495^{\circ} \mathrm{C}\right)$ and $25 \mathrm{~nm}\left(800^{\circ} \mathrm{C}\right)$.

The growth rate of the film is an important factor for commercial applications. The growth rate must be sufficiently high to ensure a viable production rate.

The growth rate of the B-NCD films prepared in this study was found to be linearly dependent on temperature in all samples, and varied by one order of magnitude between samples prepared at the lowest temperature and at the highest temperature.

The growth rate of the sample prepared at $450{ }^{\circ} \mathrm{C}$ was $40 \mathrm{~nm} /$ hour. The highest growth rate was for the sample prepared at $800^{\circ} \mathrm{C}$, where the value was over $500 \mathrm{~nm} /$ hour. The growth rates of all samples are summarized in Fig. 3.

The growth rate values at various temperatures are typical for the given methane concentration. As the growth rates are strongly dependent on methane concentration, an increase in the methane fraction during deposition could be used for boosting the growth rate $[7]$.

\section{Conclusions}

In this work, we have investigated the quality of boron-doped nanodiamond films prepared at various temperatures in order to find the optimal conditions for application in implantable electrodes. Five samples were deposited by MWPECVD for the experiment. Morphology and thickness were investigated by AFM. Raman spectroscopy was used for estimating the boron content and for determining the quality of 

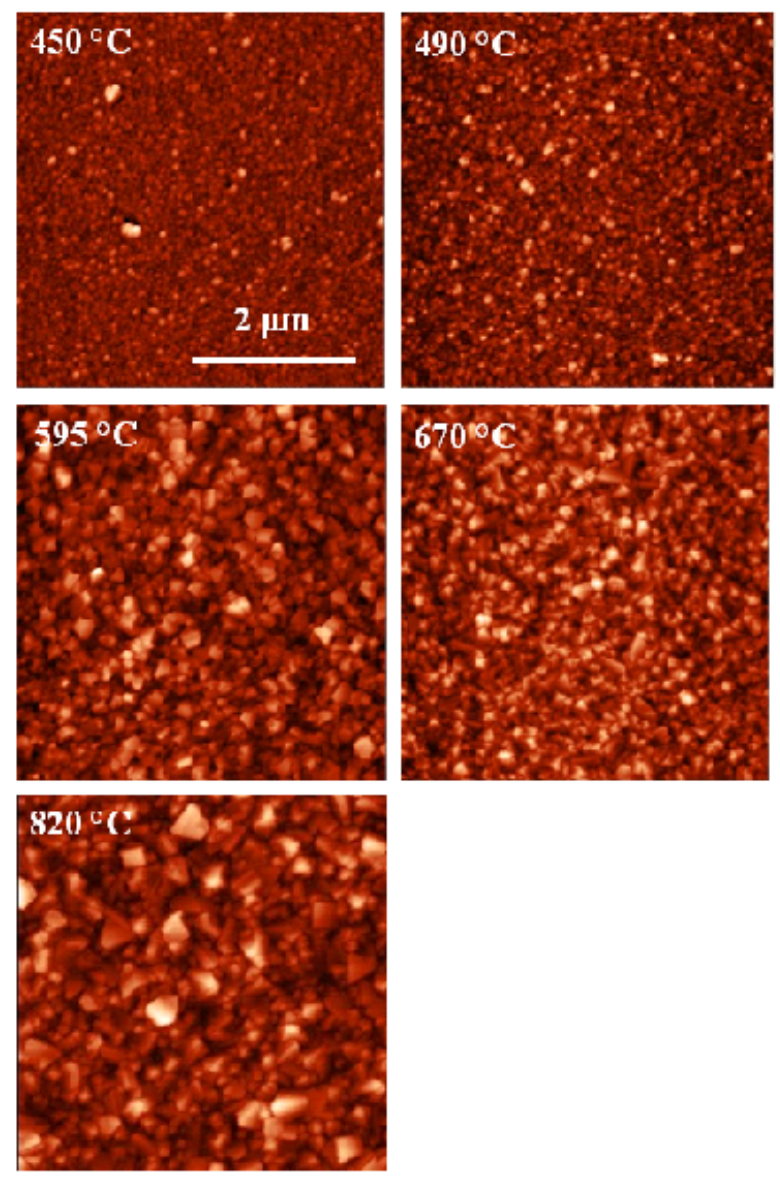

Figure 4: $5 \mu \mathrm{m} \times 5 \mu \mathrm{m}$ AFM images of B-NCD films prepared at various temperatures.

the films. We found that the level of boron doping was strongly dependent on the deposition temperature. Low temperatures, and also high temperatures, led to low boron incorporation. An optimal sample of B-NCD films was prepared at $595{ }^{\circ} \mathrm{C}$. This sample had the highest level of boron incorporation and a low amount of impurities. The growth rate achieved at this temperature is considered sufficient for potential commercial production.

\section{Acknowledgements}

The research presented in this paper was supervised by Václav Petrák, FBMI CTU in Prague. Special thanks to Vladkka Petráková from FBMI CTU in Prague for useful consultations. We are grateful to colleagues from the Institute of Physics AS CR for their help with AFM measurements, and to colleagues from the Heyrovsky Institute of Physical Chemistry AS CR for their help with Raman Spectroscopy measurements. This work was supported by the Grant Agency of the Czech Technical University in Prague, grant No. CTU $10 / 811700$.

\section{References}

[1] M. Houdek, et al. Neuromodulace. Grada, 2007.

[2] P. W. May. Diamond thin films: A 21st-century material. Philosophical Transactions of the Royal Society of London Series A Mathematical Physical and Engineering Sciences 358(1766):473-495, 2000 .

[3] P. W. May, W. J. Ludlow, M. Hannaway, et al. Raman and conductivity studies of boron-doped microcrystalline diamond, faceted nanocrystalline diamond and cauliflower diamond films. Diamond \& Related Materials 17(2):105-117, 2008.

[4] S. Myllymaa, K. Myllymaa, R. Lappalainen. Flexible implantable thin film neural electrodes. http://cdn.intechweb.org/pdfs/9218. pdf, [2012-03-14].

[5] A. Norlin, J. Pan, C. Leygraf. Investigation of Pt, $\mathrm{Ti}, \mathrm{TiN}$, and nano-porous carbon electrodes for implantable cardioverter-defibrillator applications. Electrochimica Acta 49(22-23):4011-4020, 2004.

[6] S. Prawer, R. J. Nemanich. Raman spectroscopy of diamond and doped diamond. Philosophical Transactions of the Royal Society of London Series A 362(1824):2537-2565, 2004.

[7] O.A. Williams, M. Nesladek, M. Daenen, et al. Growth, electronic properties and applications of nanodiamond. Diamond \& Related Materials 17(7-10):1080-1088, 2008. 